\title{
GEOMETRY OF ANONYMOUS BINARY SOCIAL CHOICES THAT ARE STRATEGY-PROOF
}

\author{
Achille Basile* \\ Dipartimento di Scienze Economiche e Statistiche \\ Università Federico II, 80126 Napoli, Italy \\ E-mail: basile@unina.it \\ Surekha Rao \\ School of Business and Economics \\ Indiana University Northwest, Gary, IN 46408 \\ E-mail: skrao@iun.edu \\ and \\ K. P. S. Bhaskara Rao \\ Department of Computer Information Systems \\ Indiana University Northwest, Gary, IN 46408 \\ E-mail: bkoppart@iun.edu
}

August 6, 2020

*Corresponding author. 


\begin{abstract}
Let $V$ be society whose members express preferences about two alternatives, indifference included. Identifying anonymous binary social choice functions with binary functions $f=f(k, m)$ defined over the integer triangular grid $G=\left\{(k, m) \in \mathbb{N}_{0} \times \mathbb{N}_{0}: k+m \leq|V|\right\}$, we show that every strategy-proof, anonymous social choice function can be described geometrically by listing, in a sequential manner, groups of segments of $\mathrm{G}$, of equal (maximum possible) length, alternately horizontal and vertical, representative of preference profiles that determine the collective choice of one of the two alternatives. Indeed, we show that every function which is anonymous and strategy-proof can be described in terms of a sequence of nonnegative integers $\left(q_{1}, q_{2}, \cdots, q_{s}\right)$ corresponding to the cardinalities of the mentioned groups of segments. We also analyze the connections between our present representation with another of our earlier representations involving sequences of majority quotas.

A Python code is available with the authors for the implementation of any such social choice function.
\end{abstract}

AMS Subject Classification: 91B14

JEL Code: D71

Keywords: social choice functions, anonymity, strategy-proofness, indifference, cone, comprehensive set. 


\section{Introduction}

Let $V$ be a finite society that has to implement one of two projects, say alternative $a$ or alternative $b$, based upon the profile $P=\left(P_{v}\right)_{v \in V}$ of preferences expressed by its members. The choice $P_{v}$ of individual $v$ can be either in favor of $a$, or in favor of $b$, or it can be indifference between the two alternatives 1 . To determine the collective choice corresponding to the profile $P$ of preferences, the society needs to apply a rule, or social choice function (scf, for short), we may say. Let $\phi: \mathcal{P} \rightarrow\{a, b\}$ be such a rule. Notice that we denote by $\mathcal{P}$ the set of all possible profiles.

To ensure a fair consideration of the opinions of all agents, and to prevent strategical false declarations, typical assumptions on the scf $\phi$ are anonymity and strategy-proofnes (see Definition 2.1).

This paper gives a new geometric characterization of anonymous, strategy-proof scfs, and a corresponding new representation theorem for such functions.

If $\phi$ is an anonymous scf, the collective choice $\phi(P)$ only depends upon the pair $(k(P), m(P))$ of voters that prefer $a$ and $b$, respectively. Consequently, $\phi$ can be seen as a function $f$ from the integer triangular grid: $G=\left\{(k, m) \in \mathbb{N}_{0} \times \mathbb{N}_{0}: k+m \leq|V|\right\}$, to the set $\{a, b\}$. Precisely:

$$
\phi(P)=f(k(P), m(P))
$$

is the law that gives the identification.

We show that $\phi$ is in addition strategy-proof if and only if the function $f$ satisfies the following geometric property: whenever a point $\left(k^{*}, m^{*}\right)$ of $G$ belongs to the inverse image $\{f=a\}$ of the alternative $a$, then all points belonging to the intersection of $G$ with the cone $A$ in the picture below 2 , also belong to $\{f=a\}$, namely they represent profiles that determine a as the social choice.

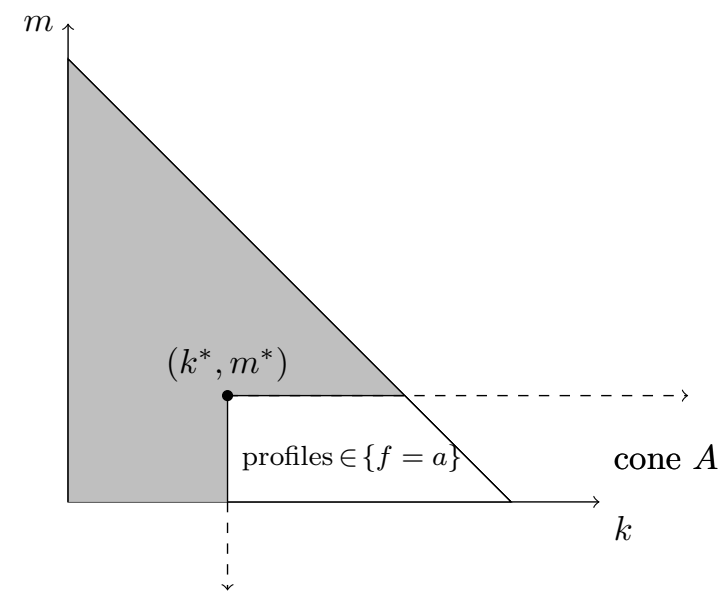

Figure $\alpha$

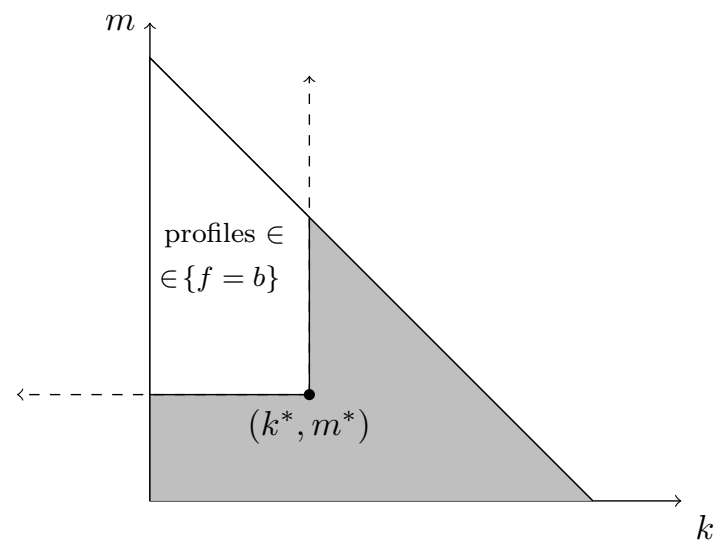

Figure $\beta$

\footnotetext{
${ }^{1}$ We can write, for short, $P_{v}=a, b, a b$, to mean $a \underset{P_{v}}{\succ} b, b \underset{P_{v}}{\succ} a$, or $a \underset{P_{v}}{\sim} b$ respectively.

${ }^{2}$ The cone $A$, of vertex $\left(k^{*}, m^{*}\right)$, is white with dashed axis in Figure $\alpha$, whereas $G$ is in gray.
} 
The above property can be formalized as the comprehensiveness of the inverse image of the alternative $a$. Comprehensiveness is a property well known for its use in the theory of Core Allocations (see Aliprantis et al. [1]). Strategy-profness of an anonymous $f$ is at the same time equivalent to the fact that the inverse image of the alternative $b$ is comprehensive with respect to the cone with vertex $\left(k^{*}, m^{*}\right)$ opposite to $A$ (see Figure $\beta$ above).

As a consequence of the above geometric characterization, we have the following representation theorem.

Theorem 1.1 The anonymous, strategy-proof scfs are all and only the functions $f: G \rightarrow$ $\{a, b\}$ that correspond to finite sequences $\boldsymbol{q}=\left(q_{1}, q_{2}, \ldots\right)$ of integers, with $q_{1} \in\{0,1, \ldots,|V|\}$, every further term $q_{i} \in\{1,2, \ldots,|V|\}$, and $\sum_{i} q_{i}=|V|+1$, such that, in order:

$q_{1}$ horizontal segments in $G$ of maximum possible length, represent profiles determining, according to $f$, the social choice $a$;

$q_{2}$ vertical segments in $G$ of maximum possible length given the above, represent profiles determining, according to $f$, the social choice $b$;

$q_{3}$ horizontal segments in $G$ of maximum possible length given the above, represent profiles determining, according to $f$, the social choice $a$;

$q_{4}$ vertical segments in $G$ of maximum possible length given the above, represent profiles determining, according to $f$, the social choice $b$;

and so on, until $G$ is filled.

The figure below illustrates the representation theorem.

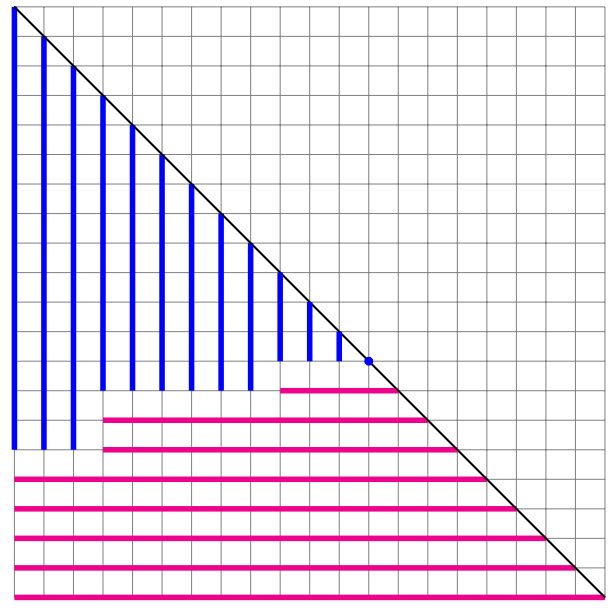

Figure 0

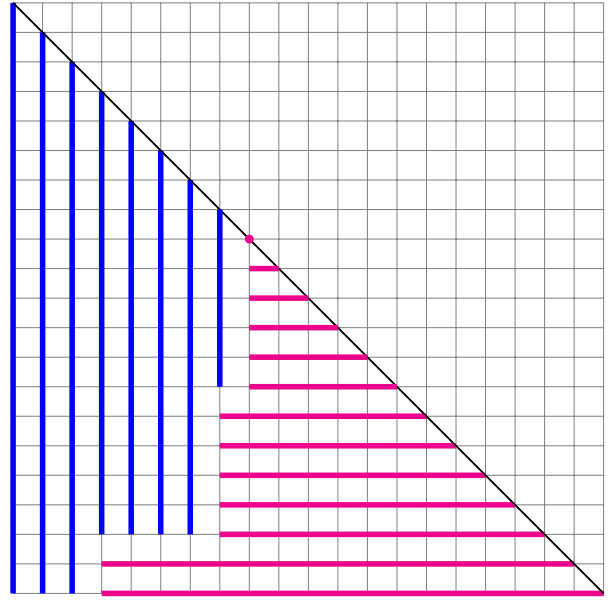

Figure $0_{b i s}$

In both figures we have $|V|=20$. Blue vertical lines represent profiles corresponding to which the social choice is $b$; the magenta horizontal lines represent profiles corresponding to 
which the social choice is $b$. In Figure 0 we have $\boldsymbol{q}=(5,3,2,6,1,4)$ and the scf represented implements the alternative $a$ if all voters are indifferent between $a$ and $b$. In Figure $0_{b i s}$ we have $\boldsymbol{q}=(0,3,2,4,5,1,6)$ and the scf represented implements the alternative $b$ if all voters are indifferent.

Given the role of the integers $q_{i}$, we give the name $\{a, b\}$-list to the sequence $\boldsymbol{q}$.

Comparison with previous literature. Despite the general circumstance according to which representation formulas are a quite obvious topic to investigate, to the best of our knowledge, only recently representation theorems of anonymous strategy-proof scfs in presence of indifference have been proposed. If indifference is not allowed, 6, Corollary of page 63] completely describes the $|V|+2$ existing anonymous strategy proof scfs. Allowing for indifference, in the literature we find [5, Theorem 2], and the representation [3, Theorem 2.7]. Anonymous strategy-proof scfs are represented in [3] by means of suitably monotone sequences (up and down sequences) $\boldsymbol{k}$ of majority quotas, in this way providing a natural extension of [6, Corollary of page 63] for representing the $2^{|V|+1}$ anonymous strategy-proof scfs that exist when agents can declare indifference.

[5, Theorem 2], [3, Theorem 2.7] and Theorem 1.1, are definitely three different results. The first two having been compared in [3], in Section 4 we compare in details the last two, also providing the conversion formulas (Theorem 4.1 and its reverse) to move back and forth from the present geometric representation to the extended quota majority representation [3. Theorem 2.7].

The remaining sections of the paper are organized as follows. In Section 2 , we set the notations and introduce dually monotone functions. By means of such functions the strategyproofness of anonymous scfs is characterized and also related to the comprehensiveness of the inverse image of the alternatives. Corollary 2.9 is an intermediate representation theorem. Section 3 is devoted to the characterization of dual monotonicity my means of $\{a, b\}$-lists. Our main result Theorem 1.1 just combines Corollary 2.9 with Theorem 3.1. The last section presents the proofs of Theorems 3.1 and 4.1 .

A Python code, written by the authors, that implements a given anonymous strategy-proof scf and shows the geometric representation is available. This code can also be used to convert proper majority quotas into the corresponding $\{a, b\}$-list and viceversa.

\section{Basic notions, notation}

Throughout the sequel we assume that the cardinality of $V$ is $n$. Given a profile $P$, the set $D(a, P)$ (resp. $D(b, P)$ ) denotes the subset of $V$ consisting of voters that prefer $a$ over $b$ (resp. $b$ over $a$ ). The agents that are indifferent are obviously those of $V \backslash[D(a, P) \cup D(b, P)]$. We also set $k(P)=|D(a, P)|$ and $m(P)=|D(b, P)|$.

Typically a scf, i.e. a function that maps profiles of preferences to alternatives that have to be implemented as the result of a collective choice, will be denoted by $\phi$. The following 
definitions are well established and widely employed in the literature after the pioneering works [4],[7]; see also [2] for specific reference to the binary case.

Definition 2.1 A scf $\phi$ is:

anonymous, if $\phi(P)=\phi(P \circ \sigma)=\phi\left(\left(P_{\sigma(v)}\right)_{v \in V}\right)$, for every profile $P$ and for every permutation $\sigma$ of $V$.

non-manipulable, if $\phi\left(P_{v}, P_{-v}\right) \succ_{P_{v}} \phi\left(Q_{v}, P_{-v}\right)$, for every voter $v$, for every profile $P$, and for every weak ordering $Q_{v}$.

This paper deals with binary scfs, i.e. only two alternatives are considered. For the sake of brevity we write scf to mean binary scf.

Let us denote by $\boldsymbol{\Phi}$ the set of all anonymous scfs $\phi$.

Let us denote by $\boldsymbol{F}$ the set of all functions $f=f(k, m)$ defined on the integer triangular grid:

$$
G=\left\{(k, m) \in[0, n]^{2}: k+m \leq n\right\},
$$

and with values $f(k, m) \in\{a, b\}$. We set $\ell:=n-k-m$.

Definition 2.2 Two profiles $P$ and $Q$ are said to be equivalent when $k(P)=k(Q)$ and $m(P)=m(Q)$. In this case we shall write $P \equiv Q$.

Definition 2.3 For every $(k, m) \in G$, by $P(k, m)$ we denote the profile where the first $\ell$ agents are indifferent between $a$ and $b$, the subsequent $k$ agents declare to prefer $a$ and the last $m$ agents declare to prefer $b$.

The proof of the next two propositions are trivial.

Proposition 2.4 The quotient $\mathcal{P} / \equiv$ and the grid $G$ are in a one-to-one correspondence by means of the map

$$
P / \equiv \mapsto(k(P), m(P)) \text {, whose inverse is }(k, m) \mapsto P(k, m) / \equiv \text {. }
$$

Moreover,

$$
P \equiv Q \Rightarrow \phi(P)=\phi(Q), \forall \phi \in \mathbf{\Phi}
$$

Proposition 2.5 The function sets $\boldsymbol{\Phi}$ and $\boldsymbol{F}$ are in a one-to-one correspondence by means of the map

$L: \phi \in \boldsymbol{\Phi} \mapsto L(\phi) \in \boldsymbol{F}$, where $L(\phi)$ is the function $f_{\phi}$ defined by $f_{\phi}(k, m)=\phi(P(k, m))$.

The inverse of $L$ is

$$
f \in \boldsymbol{F} \mapsto \phi_{f} \in \boldsymbol{\Phi} \text { where } \phi_{f}(P):=f(k(P), m(P))
$$


We now introduce the concept of dual monotonicity for functions $f(k, m)$.

Definition 2.6 We say that $f \in \boldsymbol{F}$ is dually monotone if the following implication holds true:

$$
f(k, m)=a \Rightarrow f(k+1, m)=f(k, m-1)=a .
$$

As we see below, dual monotonicity can be defined also with reference to the alternative $b$ instead of $a$. We denote by $C_{a}$ and $C_{b}$ the two cones in the $k m$-plane defined respectively by

$$
\begin{gathered}
(\alpha, \beta) \in C_{a} \Leftrightarrow \alpha \geq 0 \& \beta \leq 0, \\
(\alpha, \beta) \in C_{b} \Leftrightarrow \alpha \leq 0 \& \beta \geq 0 .
\end{gathered}
$$

If we adopt the notation $H+C$, when $H \subseteq G$ and $C$ is one of the two above cones, for the set $\left\{\left(k^{\prime}, m^{\prime}\right) \in G:\left(k^{\prime}, m^{\prime}\right)=(k, m)+(\alpha, \beta)\right.$, for some $(k, m) \in H$ and some $\left.(\alpha, \beta) \in C\right\}$, then we easily see that:

Proposition 2.7 For $f \in \boldsymbol{F}$, TFAE:

\section{1. $f$ is dually monotone}

2. $f(k, m)=b \Rightarrow f(k-1, m)=f(k, m+1)=b$

3. $\{f=a\}+C_{a} \subseteq\{f=a\}$

4. $\{f=b\}+C_{b} \subseteq\{f=b\}$

Property 3. above says that the set $\{f=a\}$ is comprehensive. Equivalently the set $\{f=b\}$ is comprehensive. Notice that, however, the cones are different.

It is obvious from the previous proposition that if $f$ is dually monotone, then $f(0, n)=a$ corresponds to the constant function $f=a$, and $f(n, 0)=b$ corresponds to the constant function $f=b$.

Theorem $2.8 \phi \in \mathbf{\Phi}$ is strategy-proof if and only if $f_{\phi}$ is dually monotone.

PROOF: We write here $f$ instead of $f_{\phi}$, for short.

Let us assume that $\phi$ is anonymous and strategy-proof. If $f$ is not dually monotone, we have $f(k, m)=a$ and either $f(k+1, m)=b$ or $f(k, m-1)=b$. In both cases we get a contradiction. We analyze the case $f(k+1, m)=b$, the other case being analogous. By definition we have $\phi(P(k, m))=a$ and $\phi(P(k+1, m))=b$. The difference between the profiles $P(k, m)$ and $P(k+1, m)$ is: one agent that in $P(k, m)$ is indifferent, under $P(k+1, m)$ prefers $a$. So, this agent can advantageously manipulate $P(k+1, m)$ by declaring indifference. 
For the converse let us suppose that $f_{\phi}$ is dually monotone whereas $\phi$ is manipulable. So, we have an agent $v_{0}$, a profile $P$, and a profile $Q=\left(Q_{v_{0}}, P_{-v_{0}}\right)$ such that

(*) $\quad \phi\left(Q_{v_{0}}, P_{-v_{0}}\right) \underset{P_{v_{0}}}{\succ} \phi\left(P_{v_{0}}, P_{-v_{0}}\right)$.

Let us set $|D(a, P)|=k,|D(b, P)|=m$ and $|D(a, Q)|=k^{\prime},|D(b, Q)|=m^{\prime}$. The relation $(*)$ can be written as

$$
(*) \quad f\left(k^{\prime}, m^{\prime}\right) \underset{P_{v_{0}}}{\succ} f(k, m),
$$

and only two cases are possible from $(*)$.

Case $v_{0}$ prefers $a$ : It means that $f\left(k^{\prime}, m^{\prime}\right)=a$. Comparing $\left(Q_{v_{0}}, P_{-v_{0}}\right)$ and $\left(P_{v_{0}}, P_{-v_{0}}\right)$, we see that if the agent $v_{0} Q_{v_{0}}$-prefers $b$, then $m^{\prime}=m+1$ and $k^{\prime}=k-1$. If the agent $v_{0}$ is $Q_{v_{0}}$-indifferent between $a$ and $b$, then $m^{\prime}=m$ and $k^{\prime}=k-1$.

In both cases the dual monotonicity of $f$ gives us that $f(k, m)=a$ and this is impossible.

Case $v_{0}$ prefers $b$ : It is analogous.

Corollary 2.9 A scf $\phi$ is anonymous and strategy-proof if and only if it is of the form $\phi_{f}$ for a dually monotone $f \in \boldsymbol{F}$.

\section{Dually monotone functions on $G$}

In this section we give a representation of all dually monotone functions. Because of Corollary 2.9, this will give us the representation of all anonymous, strategy-proof scfs that have been stated as Theorem 1.1.

Let $\boldsymbol{q}$ be a finite sequence $\left(q_{1}, q_{2}, \ldots\right)$. We say that it is an $\{a, b\}$-list if $q_{1} \in\{0,1, \ldots, n\}$, every further term $q_{i}$ belongs to $\{1,2, \ldots, n\}$, and $\sum_{i} q_{i}=n+1$. Given $\boldsymbol{q}$, we define the sets

$$
Q_{a}=\left\{\left(0, q_{1}-1\right),\left(q_{2}, q_{1}+q_{3}-1\right),\left(q_{2}+q_{4}, q_{1}+q_{3}+q_{5}-1\right), \ldots\right\}
$$

and

$$
Q_{b}=\left\{\left(q_{2}-1, q_{1}\right),\left(q_{2}+q_{4}-1, q_{1}+q_{3}\right),\left(q_{2}+q_{4}+q_{6}-1, q_{1}+q_{3}+q_{5}\right), \ldots\right\} .
$$

It is elementary to check that

$$
\left[Q_{a}+C_{a}\right] \cap\left[Q_{b}+C_{b}\right]=\varnothing
$$

hence the following $f_{\boldsymbol{q}}$ is a well defined function on $G$.

$$
(k, m) \in G \mapsto f_{\boldsymbol{q}}(k, m)= \begin{cases}a, & \text { if }(k, m) \in Q_{a}+C_{a} \\ b, & \text { if }(k, m) \in Q_{b}+C_{b} .\end{cases}
$$


The structure of the function $f_{\boldsymbol{q}}$ has been already described more intuitively in the statement of Theorem 1.1. Indeed, it is easy to check that $f_{\boldsymbol{q}}$ can be described as follows.

In order:

$q_{1}$ horizontal segments in $G$ of maximum possible length are mapped by $f_{\boldsymbol{q}}$ to $a$;

$q_{2}$ vertical segments in $G$ of maximum possible length given what above, are mapped by $f_{\boldsymbol{q}}$ to $b$;

$q_{3}$ horizontal segments in $G$ of maximum possible length given what above, are mapped by $f_{\boldsymbol{q}}$ to $a$;

$q_{4}$ vertical segments in $G$ of maximum possible length given what above, are mapped by $f_{\boldsymbol{q}}$ to $b$;

and so on.

Theorem 3.1 An element $f \in \boldsymbol{F}$ is dually monotone if and only if for some $\{a, b\}$-list $\boldsymbol{q}$ one has $f=f_{\boldsymbol{q}}$.

The proof of the theorem will be given in the Appendix.

We conclude the section rephrasing Theorem 1.1 as follows.

Theorem 1.1 $A$ scf $\phi$ is anonymous and strategy-proof if and only if $\phi=\phi_{f \boldsymbol{q}}$, for some $\{a, b\}$-list $\boldsymbol{q}$.

\section{Comparison with proper extended quota majority methods}

In [3] we proved that the scfs that are anonymous and strategy-proof can be characterized as proper extended quota majority methods (denoted by $\phi_{\boldsymbol{k}}$; see [3, Definition 2.2] for the definition of the $\left.\operatorname{scf} \phi_{\boldsymbol{k}}\right)$, the majority quotas $\boldsymbol{k}=\left(k_{0}, k_{1}, \ldots, k_{r}\right)$ forming a sequence satisfying the following up and down conditions.

down-up:

$$
\begin{aligned}
& \begin{array}{|c|l|c|}
\hline 0 & <k_{r-1}<\ldots<k_{5}<k_{3}<k_{1}<k_{0}<k_{2}<k_{4}<k_{6}<\ldots< & n+1=k_{r} \\
\hline
\end{array} \\
& \begin{array}{|l|l|l|}
\hline 0=k_{r} & <\ldots<k_{5}<k_{3}<k_{1}<k_{0}<k_{2}<k_{4}<k_{6}<\ldots<k_{r-1}< & n+1 \\
\hline
\end{array}
\end{aligned}
$$

up-down:

$$
\begin{array}{|c|c|c|}
\hline n+1=k_{r} & >\ldots>k_{5}>k_{3}>k_{1}>k_{0}>k_{2}>k_{4}>k_{6}>\ldots>k_{r-1}> & 0 \\
\hline
\end{array}
$$


At the same time, as we have seen in Theorem 1.1, the scfs under consideration are identifiable by means of $\{a, b\}$-lists $\boldsymbol{q}$ as scfs $\phi=\phi_{f_{\boldsymbol{q}}}$. It is therefore natural to investigate formulas that connect up and down majority quotas $\boldsymbol{k}$ and $\{a, b\}$-lists $\boldsymbol{q}$ when they identify the same scf, namely when $\phi_{\boldsymbol{k}}=\phi_{f_{\boldsymbol{q}}}$.

The purpose of the present section is to provide a one-to-one map $T$ that transforms an up and down sequence $\boldsymbol{k}$ into an $\{a, b\}$-list $\boldsymbol{q}$ such that the scf represented is the same.

It will be a map like

$$
\left(q_{1}, q_{2}, \ldots, q_{s}\right)=T\left(k_{0}, k_{1}, \ldots, k_{r}\right)
$$

i.e. we shall give formulas like

$$
q_{i}=T_{i}\left(k_{0}, k_{1}, \ldots, k_{r}\right), \quad i \leq s=s(r)
$$

Theorem 4.1 Let $\phi$ be an anonymous, strategy-proof scf and $\boldsymbol{k}=\left(k_{0}, k_{1}, \ldots, k_{r}\right)$ the corresponding up and down sequence of majority quotas (i.e. $\phi=\phi_{\boldsymbol{k}}$ ).

1. If $\phi$ selects the alternative a when the collectivity is unanimously indifferent between the two alternatives $a$ and 3 , the $\{a, b\}$-list satisfying the equation $\phi_{\boldsymbol{k}}=\phi_{f_{\boldsymbol{q}}}$ is $\left(q_{1}, q_{2}, \ldots, q_{r}, q_{r+1}\right)$, where

$$
\begin{gathered}
q_{1}=k_{r-1}^{\circ}, q_{2}=k_{r-2}, q_{3}=k_{r-3}^{\circ}-k_{r-1}^{\circ}, q_{4}=k_{r-4}-k_{r-2}, \ldots \\
q_{i}=\left\{\begin{array}{ll}
k_{r-i}^{\circ}-k_{r-i+2}^{\circ}, & \text { if } 5 \leq i \leq r \text { is odd } \\
k_{r-i}-k_{r-i+2}, & \text { if } 5 \leq i \leq r \text { is even },
\end{array} \quad q_{r+1}=n+1-\sum_{1}^{r} q_{i}\right.
\end{gathered}
$$

2. If $\phi$ selects the alternative $b$ when the collectivity is unanimously indifferent between the two alternatives $a$ and $b$, the $\{a, b\}$-list satisfying the equation $\phi_{\boldsymbol{k}}=\phi_{f_{\boldsymbol{q}}}$ is $\left(0, q_{1+1}, \ldots, q_{1+r}, q_{r+2}\right)$, where

$$
\begin{gathered}
q_{1+1}=k_{r-1}, q_{1+2}=k_{r-2}^{\circ}, q_{1+3}=k_{r-3}-k_{r-1}, q_{1+4}=k_{r-4}^{\circ}-k_{r-2}^{\circ}, \ldots \\
q_{1+i}=\left\{\begin{array}{ll}
k_{r-i}-k_{r-i+2}, & \text { if } 5 \leq i \leq r \text { is odd } \\
k_{r-i}^{\circ}-k_{r-i+2}^{\circ}, & \text { if } 5 \leq i \leq r \text { is even },
\end{array} \quad q_{r+2}=n+1-\sum_{1}^{r} q_{1+i}\right.
\end{gathered}
$$

The proof of the theorem will be given in the Appendix.

Above formulas can be both written as $\boldsymbol{x}=\boldsymbol{T} \boldsymbol{y}$ if $\boldsymbol{T}$ is the lower triangular matrix of order $r$ :

\footnotetext{
${ }^{3}$ We recall that, by the definition of extended quota majority method, $k_{r}=0\left(\right.$ resp. $\left.k_{r}=n+1\right)$ corresponds to scfs choosing the alternative $a$ (resp. $b$ ) for a collectivity which is unanimously indifferent between the two alternatives $a$ and $b$.
} 


$$
\boldsymbol{T}=\left[\begin{array}{cccccccccccccc}
1 & 0 & 0 & 0 & 0 & 0 & 0 & 0 & 0 & 0 & 0 & 0 & 0 & 0 \\
0 & 1 & 0 & 0 & 0 & 0 & 0 & 0 & 0 & 0 & 0 & 0 & 0 & 0 \\
-1 & 0 & 1 & 0 & 0 & 0 & 0 & 0 & 0 & 0 & 0 & 0 & 0 & 0 \\
0 & -1 & 0 & 1 & 0 & 0 & 0 & 0 & 0 & 0 & 0 & 0 & 0 & 0 \\
0 & 0 & -1 & 0 & 1 & 0 & 0 & 0 & 0 & 0 & 0 & 0 & 0 & 0 \\
0 & 0 & 0 & -1 & 0 & 1 & 0 & 0 & 0 & 0 & 0 & 0 & 0 & 0 \\
& & & & & & & & & & & & & \\
& & & & & \cdots & \cdots & \cdots & & & & & & \\
0 & 0 & 0 & 0 & 0 & 0 & 0 & 0 & 0 & -1 & 0 & 1 & 0 & 0 \\
0 & 0 & 0 & 0 & 0 & 0 & 0 & 0 & 0 & 0 & -1 & 0 & 1 & 0 \\
0 & 0 & 0 & 0 & 0 & 0 & 0 & 0 & 0 & 0 & 0 & -1 & 0 & 1
\end{array}\right]
$$

and, for formula 1. of Theorem 4.1,

$$
\boldsymbol{x}^{\prime}=\left(q_{1}, q_{2}, \ldots, q_{r}\right), \text { and } \boldsymbol{y}^{\prime}=\left(k_{r-1}^{\circ}, k_{r-2}, k_{r-3}^{\circ}, \ldots, k_{r-(r-1)}^{\circ}, k_{r-r}\right) .
$$

For 2. of Theorem 4.1, we have to consider

$$
\boldsymbol{x}^{\prime}=\left(q_{1+1}, q_{1+2}, \ldots, q_{1+r}\right), \text { and } \boldsymbol{y}^{\prime}=\left(k_{r-1}, k_{r-2}^{\circ}, k_{r-3}, \ldots, k_{r-(r-1)}, k_{r-r}^{\circ}\right) .
$$

By using the inverse of $\boldsymbol{T}$

$$
\boldsymbol{T}^{-1}=\left[\begin{array}{cccccccccccccc}
1 & 0 & 0 & 0 & 0 & 0 & 0 & 0 & 0 & 0 & 0 & 0 & 0 & 0 \\
0 & 1 & 0 & 0 & 0 & 0 & 0 & 0 & 0 & 0 & 0 & 0 & 0 & 0 \\
1 & 0 & 1 & 0 & 0 & 0 & 0 & 0 & 0 & 0 & 0 & 0 & 0 & 0 \\
0 & 1 & 0 & 1 & 0 & 0 & 0 & 0 & 0 & 0 & 0 & 0 & 0 & 0 \\
1 & 0 & 1 & 0 & 1 & 0 & 0 & 0 & 0 & 0 & 0 & 0 & 0 & 0 \\
0 & 1 & 0 & 1 & 0 & 1 & 0 & 0 & 0 & 0 & 0 & 0 & 0 & 0 \\
& & & & & & & & & & & & & \\
& & & & & \ldots & \ldots & \ldots & & & & & & \\
0 & 1 & 0 & 1 & 0 & 1 & 0 & 1 & 0 & 1 & 0 & 1 & 0 & 0 \\
1 & 0 & 1 & 0 & 1 & 0 & 1 & 0 & 1 & 0 & 1 & 0 & 1 & 0 \\
0 & 1 & 0 & 1 & 0 & 1 & 0 & 1 & 0 & 1 & 0 & 1 & 0 & 1
\end{array}\right]
$$

we solve, with respect to $\boldsymbol{k}$, given $\boldsymbol{q}$, the equation $\phi_{\boldsymbol{k}}=\phi_{f_{\boldsymbol{q}}}$ as follows.

Theorem 4.1 reversed Let $\phi$ be an anonymous, strategy-proof scf and $\boldsymbol{q}=\left(q_{1}, \ldots, q_{s}\right)$ the corresponding $\{a, b\}$-list (i.e. $\left.\phi=\phi_{f \boldsymbol{q}}\right)$.

1. If $\phi$ selects the alternative a when the collectivity is unanimously indifferent between the two alternatives $a$ and $\sqrt{4}$, the sequence of majority quotas satisfying the equation $\phi_{\boldsymbol{k}}=\phi_{f \boldsymbol{q}}$ is $\left(k_{0}, k_{1}, \ldots, k_{r}\right)$, where $r=s-1$,

$$
\left(k_{r-1}^{\circ}, k_{r-2}, k_{r-3}^{\circ}, \ldots, k_{r-(r-1)}^{\circ}, k_{r-r}\right)^{\prime}=\boldsymbol{T}^{-1}\left(q_{1}, \ldots, q_{r}\right)^{\prime} \text { and } k_{r}=0
$$

\footnotetext{
${ }^{4}$ Hence $q_{1}>0$.
} 
2. If $\phi$ selects the alternative $b$ when the collectivity is unanimously indifferent between the two alternatives $a$ and $b_{5}$, the sequence of majority quotas satisfying the equation $\phi_{\boldsymbol{k}}=\phi_{f_{\boldsymbol{q}}}$ is $\left(k_{0}, k_{1}, \ldots, k_{r}\right)$, where $r=s-2$,

$$
\left(k_{r-1}, k_{r-2}^{\circ}, k_{r-3}, \ldots, k_{r-(r-1)}, k_{r-r}^{\circ}\right)^{\prime}=\boldsymbol{T}^{-1}\left(q_{1+1}, \ldots, q_{1+r}\right)^{\prime} \text { and } k_{r}=n+1
$$

Explicitly, we have

- for case 1 .

$k_{r-1}^{\circ}=q_{1}, \quad k_{r-2}=q_{2}, \quad k_{r-3}^{\circ}=q_{1}+q_{3}, \quad k_{r-4}=q_{2}+q_{4}, \quad k_{r-5}^{\circ}=q_{1}+q_{3}+q_{5}$, $k_{r-6}=q_{2}+q_{4}+q_{6}, \ldots$

- for case 2 .

$k_{r-1}=q_{1+1}, \quad k_{r-2}^{\circ}=q_{1+2}, \quad k_{r-3}=q_{1+1}+q_{1+3}, \quad k_{r-4}^{\circ}=q_{1+2}+q_{1+4}, \quad k_{r-5}=q_{1+1}+$ $q_{1+3}+q_{1+5}, k_{r-6}^{\circ}=q_{1+2}+q_{1+4}+q_{1+6}, \ldots$

\section{Appendix: proofs}

\subsection{Proof of Theorem 3.1}

Suppose $f$ is given and it is dually monotone. Let $q_{1}$ be the cardinality of the set

$$
\{m \in[0, n]: f(0, m)=a\} .
$$

In case $q_{1}=n+1$, this means $f(0, n)=a$ and because of dual monotonicity, $f=f_{\left(q_{1}\right)}$. Suppose instead that $q_{1}<n+1$. Since $f$ is dually monotone,

$$
q_{1}-1=\max \{m \in[0, n]: f(0, m)=a\} 6
$$

hence we have $f(0, m)=a, \forall m \in\left[0, q_{1}\left[\right.\right.$ and $f\left(0, q_{1}\right)=b$, and by dual monotonicity $f\left(0, q_{1}-1\right)+C_{a} \subseteq\{f=a\}$. The following figure, where magenta lines indicate points of $G$ that $f$ maps to $a$, illustrates the situation.

\footnotetext{
${ }^{5}$ Hence $\boldsymbol{q}=\left(0, q_{1+1}, \ldots, q_{1+r}, q_{r+2}\right)$.

${ }^{6}$ The elements of the set $\{m \in[0, n]: f(0, m)=a\}$, and of similar sets introduced later, are necessarily consecutive.
} 


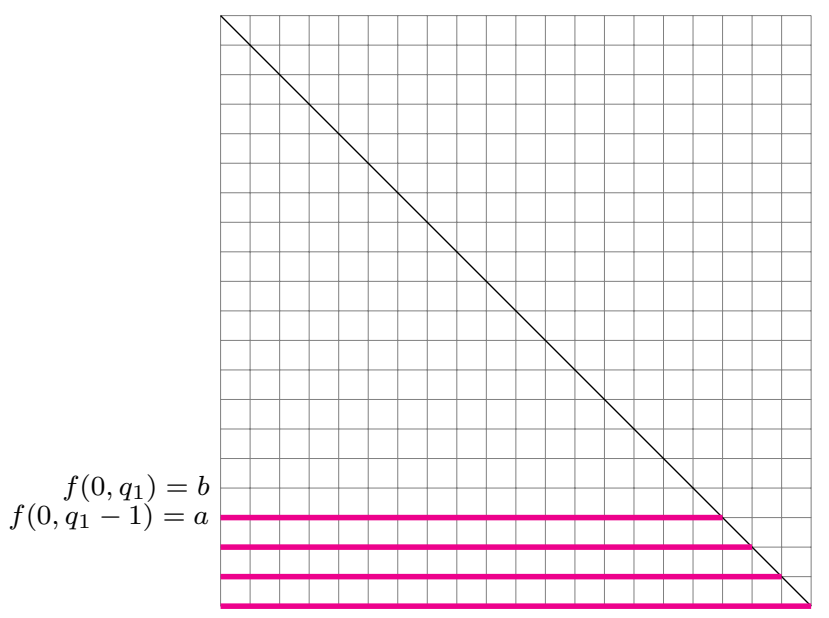

Figure 1

Now, let $q_{2}$ be the cardinality of the set

$$
\left\{k \in\left[0, n-q_{1}\right]: f\left(k, q_{1}\right)=b\right\},
$$

or, in other words, let $q_{2}-1$ be the maximum of such set. In case $q_{2}-1=n-q_{1}$, we have $\left(n-q_{1}, q_{1}\right)+C_{b} \subseteq\{f=b\}$ since $f$ is dually monotone. Hence $f=f_{\left(q_{1}, q_{2}\right)}$.

If instead we have $q_{2}-1<n-q_{1}$, then $f\left(k, q_{1}\right)=b, \forall k \in\left[0, q_{2}\left[\right.\right.$, and $f\left(q_{2}, q_{1}\right)=a$. Moreover $\left(q_{2}-1, q_{1}\right)+C_{b} \subseteq\{f=b\}$ since $f$ is dually monotone. Figure 2 extends the previous one, and illustrates the situation. The blue lines indicate points of $G$ that $f$ maps to $b$

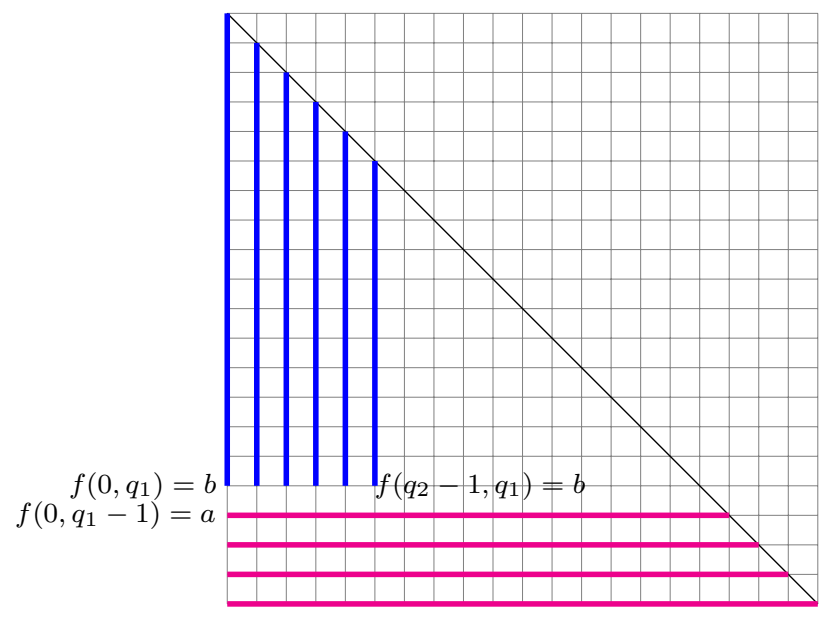

Figure 2

Let $q_{3}$ be the cardinality of the set

$$
\left\{m \in\left[q_{1}, n-q_{2}\right]: f\left(q_{2}, m\right)=a\right\},
$$


or, in other words, let $q_{1}+q_{3}-1$ be the maximum of such set. Again we have two cases. If $q_{1}+q_{3}-1=n-q_{2}$, then $\left(q_{2}, n-q_{2}\right)+C_{a} \subseteq\{f=a\}$, hence $f=f_{\left(q_{1}, q_{2}, q_{3}\right)}$.

If $q_{1}+q_{3}-1<n-q_{2}$, then $f\left(q_{2}, m\right)=a, \forall m \in\left[q_{1}, q_{1}+q_{3}-1\right]$, and $f\left(q_{2}, q_{1}+q_{3}\right)=b$. Moreover $\left(q_{2}, q_{1}+q_{3}-1\right)+C_{a} \subseteq\{f=a\}$.

We illustrate the situation with the next update of Figure 2.

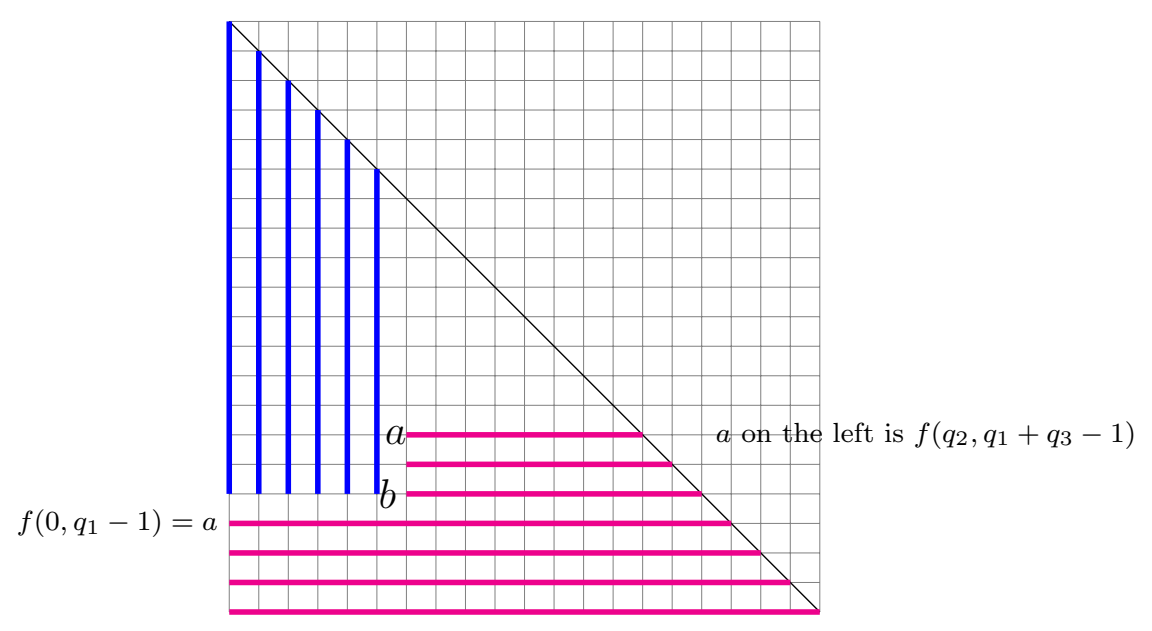

Figure 3

Let $q_{4}$ be the cardinality of the set

$$
\left\{k \in\left[q_{2}, n-q_{1}-q_{3}\right]: f\left(k, q_{1}+q_{3}\right)=b\right\},
$$

or, in other words, let $q_{2}+q_{4}-1$ be the maximum of such set.

Again we have two cases. If $q_{2}+q_{4}-1=n-q_{1}-q_{3}$, then $\left(n-q_{1}-q_{3}, q_{1}+q_{3}\right)+C_{b} \subseteq\{f=b\}$, hence $f=f_{\left(q_{1}, q_{2}, q_{3}, q_{4}\right)}$.

If $q_{2}+q_{4}-1<n-q_{1}-q_{3}$, then $f\left(k, q_{1}+q_{3}\right)=b, \forall k \in\left[q_{2}, q_{2}+q_{4}-1\right]$, and $f\left(q_{2}+q_{4}, q_{1}+q_{3}\right)=a$. Moreover $\left(q_{2}+q_{4}-1, q_{1}+q_{3}\right)+C_{b} \subseteq\{f=b\}$.

We illustrate the situation with the next update of the previous figures. 


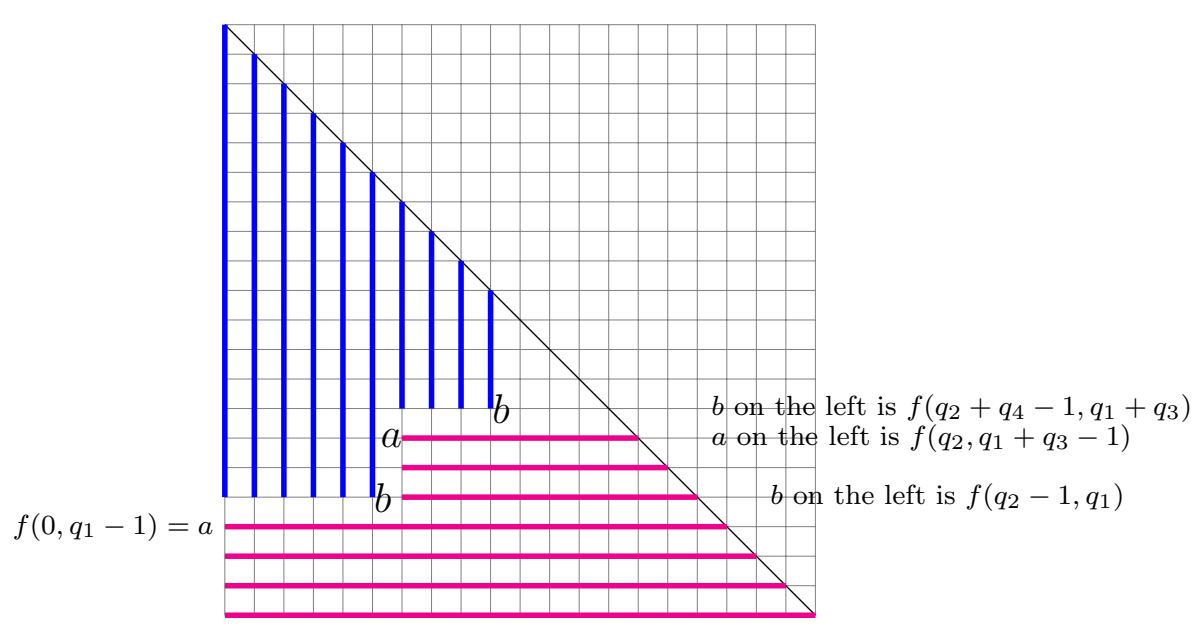

Figure 4

The procedure continues by defining $q_{5}$ as the cardinality of the set

$$
\left\{m \in\left[q_{1}+q_{3}, n-q_{2}-q_{4}\right]: f\left(q_{2}+q_{4}, m\right)=a\right\},
$$

or, in other words, $q_{1}+q_{3}+q_{5}-1=\max \left\{m \in\left[q_{1}+q_{3}, n-q_{2}-q_{4}\right]: f\left(q_{2}+q_{4}, m\right)=a\right\}$, and so on. The procedure stops as soon as the sum of the $q_{i}$ 's reaches $n+1$.

That every $f_{\boldsymbol{q}}$ is dually monotone is obvious by definition.

\subsection{Proof of Theorem 4.1}

We show the assertion in 2., assuming that the given sequence of majority quotas is

$$
\begin{array}{|l|l|l|l|}
\hline 0 & <k_{r-1}<\ldots<k_{5}<k_{3}<k_{1}<k_{0}<k_{2}<k_{4}<k_{6}<\ldots< & n+1=k_{r} \\
\hline
\end{array}
$$

in the other cases the argument is quite similar. The straightforward application of the definition of extended quota majority method (see [3, Definition 2.2]), and the fact that for the dual quotas $k_{i}^{\circ}$ the above reciprocal ordering is reversed, leads to identify that, with reference to the grid $G$,

- the profiles for which $\phi$ selects $a$ as social choice are those that corresponds to points of coordinates $(k, m)$ such that:

$k \geq k_{0}$, or $(k, m) \in\left[k_{1}, k_{0}\left[\times\left[0, k_{0}^{\circ}\left[\right.\right.\right.\right.$, or $(k, m) \in\left[k_{3}, k_{1}\left[\times\left[0, k_{2}^{\circ}[\right.\right.\right.$, or $(k, m) \in\left[k_{5}, k_{3}\left[\times\left[0, k_{4}^{\circ}\left[\right.\right.\right.\right.$, or, $\ldots,(k, m) \in\left[k_{r-1}, k_{r-3}\left[\times\left[0, k_{r-2}^{\circ}[\right.\right.\right.$.

- the profiles for which $\phi$ selects $b$ as social choice are those that corresponds to points of coordinates $(k, m)$ such that:

$m \geq k_{0}^{\circ}$, or $(k, m) \in\left[0, k_{1}\left[\times\left[k_{2}^{\circ}, k_{0}^{\circ}\left[\right.\right.\right.\right.$, or $(k, m) \in\left[0, k_{3}\left[\times\left[k_{4}^{\circ}, k_{2}^{\circ}[\right.\right.\right.$, or $(k, m) \in\left[0, k_{5}\left[\times\left[k_{6}^{\circ}, k_{4}^{\circ}\left[\right.\right.\right.\right.$, or, $\ldots,(k, m) \in\left[0, k_{r-1}\left[\times\left[k_{r}^{\circ}, k_{r-2}^{\circ}[\right.\right.\right.$. 
Since $k_{r}^{\circ}=0$, there are no full horizontal lines identifying profiles for which the social choice is $a$ (in other words $q_{1}=0$ ), whereas there are $k_{r-1}$ full vertical lines of profiles for which the social choice is $b$. So, $q_{2}=k_{r-1}$. This is illustrated in Figure 5 where $|V|=20, k_{r}=21, k_{r-1}=3, k_{r-2}=19, k_{r-3}=7, k_{r-4}=14, \ldots$

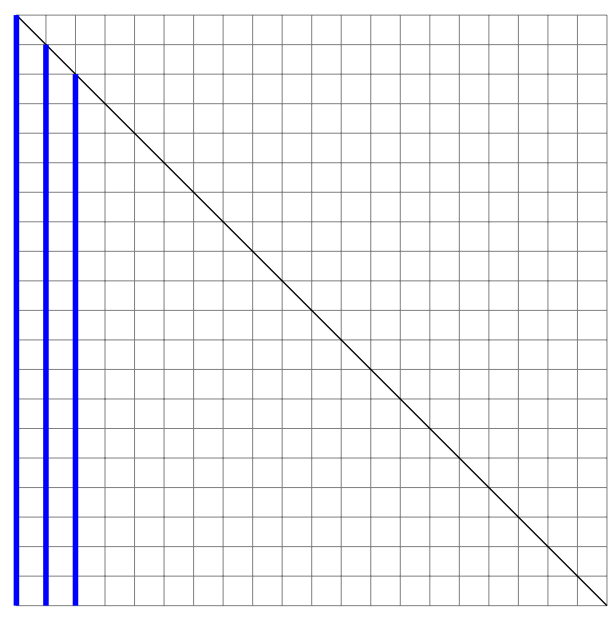

Figure 5

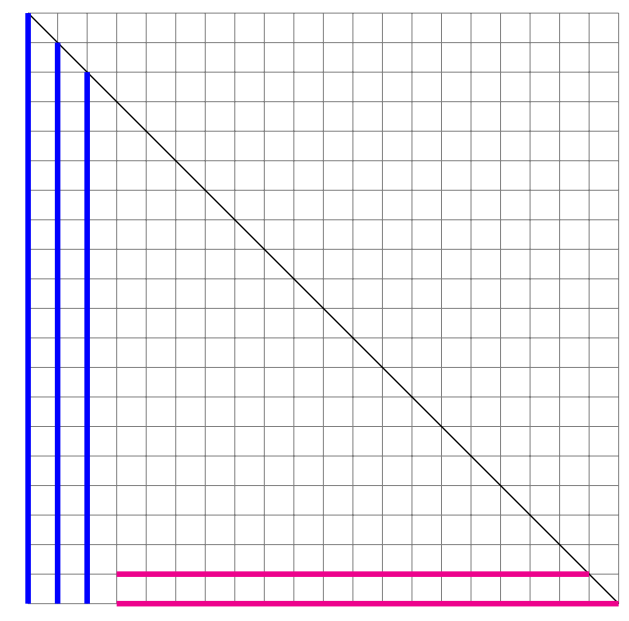

Figure 6

Now, horizontal lines that represent profiles for which the social choice is $a$ will depart from $k=k_{r-1}$ and there are $k_{r-2}^{\circ}$ many of such lines, i.e. $q_{3}=k_{r-2}^{\circ}$. This is illustrated in Figure 6

Now, further vertical lines that represent profiles for which the social choice is $b$ will depart from $k=k_{r-2}^{\circ}$ and there are $k_{r-3}-k_{r-1}$ many of such new lines, i.e. $q_{4}=k_{r-3}-k_{r-1}$. This is illustrated in Figure 7.

Again, further horizontal lines that represent profiles for which the social choice is $a$ will depart from $k=k_{r-3}$ and there are $k_{r-4}^{\circ}-k_{r-2}^{\circ}$ many of such new lines, i.e. $q_{5}=k_{r-4}^{\circ}-k_{r-2}^{\circ}$. This is illustrated in Figure 8.

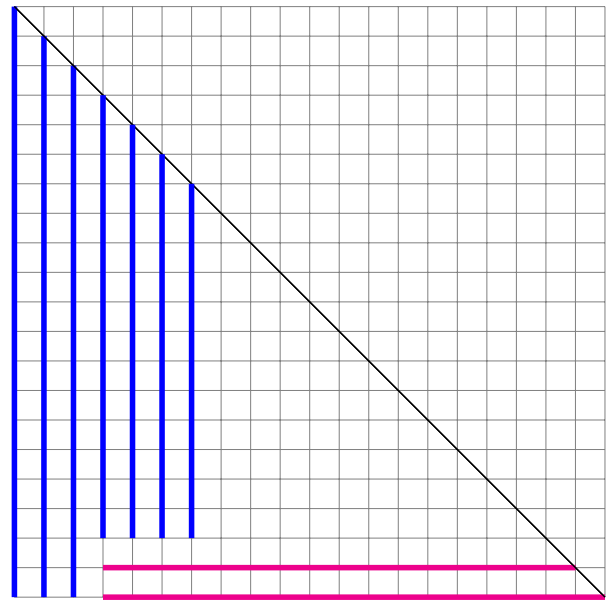

Figure 7

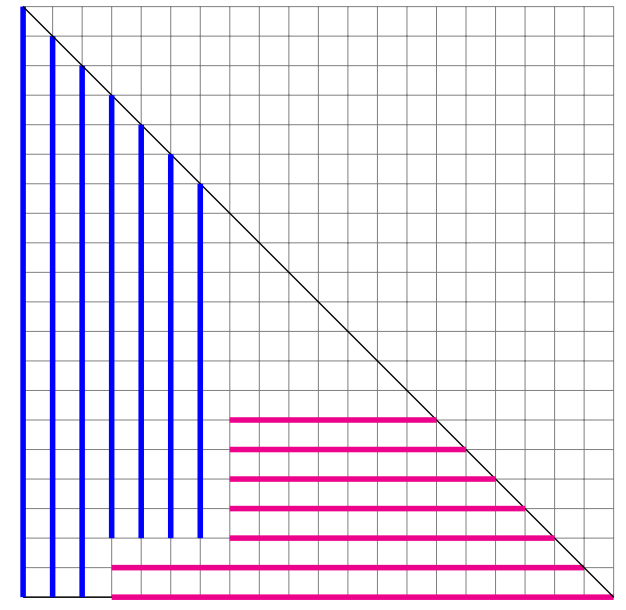

Figure 8 
The argument flows like above, alternating horizontal and vertical lines, till the grid $G$ is completely filled, according to the sequence $\boldsymbol{k}=(\ldots, 14,7,19,3,21)^{7}$.

\section{References}

[1] Aliprantis, C.D., Brown D. J. and Border, K.C., Existence and optimality of competitive equilibria, Springer-Verlag, 1990, Berlin Heidelberg.

[2] Barberà, S., Berga, D. and Moreno, B., Group strategy-proof social choice functions with binary ranges and arbitrary domains: characterization results, Int. J. Game Theory, 41(2012), 791-808.

[3] Basile, A., Rao, S., and Bhaskara Rao, K.P.S., Anonymous, non-manipulable, binary social choice, arXiv:2007.01552, (2020).

[4] Gibbard, A., Manipulation of voting schemes: a general result, Econometrica, 41(1973), 587-601.

[5] Lahiri, A. and Pramanik, A., On Strategy-proof Social Choice between Two Alternatives, Soc Choice Welf (2019). https://doi.org/10.1007/s00355-019-01220-7

[6] Moulin, H., The Strategy of Social Choice, North-Holland Publishing Company, 1983, Amsterdam.

[7] Satterthwaite, M.A., Strategy-proofness and Arrow's conditions: existence and correspondence theorems for voting procedures and social welfare functions, J. Econ. Theory, 10(1975), $187-217$.

\footnotetext{
${ }^{7}$ Observe that Figure $0_{b i s}$ is one of the possible completion of Figure 8. Precisely the one corresponding to $\boldsymbol{k}=(8,14,7,19,3,21)$.
} 